\title{
The Effect of Serum Carrying Total Flavonoids of Rhizoma Drynariae on the Proliferation and Apoptosis of Fibroblast-Like Synoviocytes in Rheumatoid Arthritis
}

\author{
Xie Jun ${ }^{1,2}$, Ling Yun², Shaohua Li ${ }^{1 *}$, Lianbo Xiao'*, Gao Chenxing ${ }^{2}$ and Zhong Sheng ${ }^{2}$ \\ ${ }^{1}$ Department of Orthopedics, The Tenth People's Hospital Affiliated to Nanjing Medical University, China \\ ${ }^{2}$ Department of Joint Orthopedics, Guanghua Hospital Affiliated to Shanghai University of Traditional Chinese Medicine, \\ China
}

*Corresponding author: Shaohua Li, Department of Orthopedics, The Tenth Hospital Affiliated to Nanjing Medical University, China Lianbo Xiao, Department of Joint Surgery, Guanghua Hospital Affiliated to Shanghai University of Traditional Chinese Medicine, China

\section{ARTICLE INFO \\ Received: 幽 September 03, 2019 \\ Published: 幽 September 16, 2019}

Citation: Bethany Malone. Developing the Role of Prehabilitation in Elective Colorectal Surgery. Biomed J Sci \& Tech Res 21(3)-2019. BJSTR. MS.ID.003594.

Keywords: Rheumatoid Arthritis; Synovial Inflammation; Fibroblast; Rhizoma Drynariae

Abbreviations: RA: Rheumatoid Arthritis; FLSs: Fibroblast-Like Synoviocytes; ELISA: Enzyme-Linked Immunosorbent Assay; DMSO: Dimethyl Sulfoxide; PCNA: Proliferating Cell Nuclear Antigen

\section{ABSTRACT}

Objective: To investigate the mechanism of the effects of total flavonoids of Rhizoma Drynariae in different concentrations on synovial inflammation in rheumatoid arthritis, which is expected to provide experimental evidence for clinical application.

Methods: Serum carrying total flavonoids of Rhizoma Drynariae was used to treat the in vitro culture of synovial cells isolated from patients with rheumatoid arthritis. Cells were identified and passaged before the cell treatment. The experimental groups included the $20 \%$ blank serum group, the $20 \%$ high-dose total flavonoids of Rhizoma Drynariae medicated serum group, 20\% low-dose total flavonoids of Rhizoma Drynariae medicated serum group, and the $20 \%$ tripterygium glucosides medicated serum group. With the serum carrying tripterygium glycosides as a positive control, the drug-carrying serum at a concentration of $20 \%$ was added to the third-generation synovial cells, and the cells were continuously cultured.

Results: Compared with the blank control serum, the sera carrying total flavonoids of Rhizoma Drynariae could significantly inhibit the expression of proliferating cell nuclear antigen (PCNA) mRNA and Bcl-2 mRNA in fibroblast-like synoviocytes (FLSs) $(\mathrm{P}<0.0001)$, and the effect of the treatment at a high dose was more significant.

Conclusion: Total flavonoids of Rhizoma Drynariae may play a role in inhibiting the proliferation of FLSs by regulating the expression of PCNA mRNA and Bcl-2 mRNA in FLSs.

\section{Introduction}

Rheumatoid arthritis (RA) is a common chronic systemic autoimmune disease. Currently, it is generally believed that the dysfunction caused by RA, cartilage damage, and bone invasion are closely related to the lack of apoptosis of fibroblast-like synoviocytes (FLSs), and insufficient apoptosis is an important cause of most tumors and autoimmune diseases [1]. Therefore, inhibition of the apoptosis of FLSs can effectively treat RA [2]. Drynaria is the dry rhizome, which is belonged to epiphytic fern, keel subject.
Drynaria is also named as monkey ginger or turmeric. The efficacy is for reinforcing kidney, strengthening bone and promoting blood circulation and removing blood stasis. Previous studies have shown that total flavonoids of Rhizoma Drynariae have anti inflammatory and osteogenesis effects [3]. However, the effect of total flavonoids of Rhizoma Drynariae on the treatment of RA is rarely reported. In this study, the effect of total flavonoids of Rhizoma Drynariae on RA was observed. 


\section{Materials and Methods}

\section{Materials}

Experimental Animals and Synovial Tissues: Twenty SPFgrade female Wistar rats $(200 \pm 10 \mathrm{~g})$ were purchased from Shanghai Experimental Animal Center of the Chinese Academy of Sciences; the license number of animal use was SYXK (Shanghai) 20080050. The synovial tissue was obtained from the Department of Joint Surgery of Guanghua Hospital affiliated with Shanghai University of Traditional Chinese Medicine. All patients were female, with a mean age of $48.23 \pm 5.36$ years and a disease duration of $7 \pm 11.45$ years. The diagnosis was in accordance with the diagnostic criteria for RA in ACR/EULAR in 2009. This study was reviewed and approved by the Ethics Committee of Guanghua Hospital affiliated with Shanghai University of Traditional Chinese Medicine.

Main Drugs: The following drugs were used in this study: total flavonoids of Rhizoma Drynariae (Qianggu Capsule, Specifications: $0.25 \mathrm{~g} \times 12$, Beijing Qihuang Pharmaceutical Co., Ltd.) and tripterygium glycoside tablets (Specifications: $10 \mathrm{mg} / \mathrm{tablet}$, Huangshi Feiyun Pharmaceutical Co., Ltd.).

\section{Methods}

Preparation of Drug-Carrying Serum: Twenty Wistar rats were randomly divided into the blank control group (gavage with sterilized water), the high-dose total flavonoids of Rhizoma Drynariae group, the low-dose total flavonoids of Rhizoma Drynariae group, and the tripterygium glycosides group. The rats in the blank control group were gavaged with sterilized water $(2 \mathrm{~mL} / \mathrm{rat} /$ day) For the rats in the total flavonoids of Rhizoma Drynariae groups, according to the clinical equivalent dose conversion equation proposed by the US FDA, Qianggu Capsule was administered at $300 \mathrm{mg} / \mathrm{kg} /$ day (containing approximately $240 \mathrm{mg} / \mathrm{kg} /$ day of total flavonoids of Rhizoma Drynariae). The total flavonoids of Rhizoma Drynariae were dissolved in sterile water to prepare a $120 \mathrm{mg} /$ $\mathrm{mL}$ solution. The low-dose group was given $0.5 \mathrm{~mL}$ per rat per day, diluted to $2 \mathrm{~mL}$ for gavage, and the high-dose group was given $2 \mathrm{~mL}$ per day per rat. The tripterygium glycoside tablets $(1.2 \mathrm{mg}$ per rat per day) were dissolved in water for gavage. All animals were administered the tablets for three consecutive days, and blood was collected 1 hour after the last administration. The blood was placed in a sterile test tube without an anticoagulant to allow natural coagulation. All tubes were placed in a test tube rack, incubated in a $37^{\circ} \mathrm{C}$ water bath for $1 \mathrm{~h}$, and centrifuged at $3000 \mathrm{r} / \mathrm{min}$ for $15 \mathrm{~min}$ to separate the serum, which was placed in another sterile test tube and stored in $\mathrm{a}-20^{\circ} \mathrm{C}$ refrigerator for later use. Just before use, the serum was inactivated at $56^{\circ} \mathrm{C}$ for $30 \mathrm{~min}$, diluted with the serumfree medium and sterilized by filtration.

Isolation, Culture and Passage of Primary Synovial Cells: Under sterile conditions, the synovial tissue was collected, placed in a 15-mL centrifuge tube containing 5\% FBS DMEM, and placed in a sterile Petri dish containing DMEM within 2 hours. Fat and blood clots on the surface were removed under sterile conditions, and the tissue was then washed 3 times. The synovial tissue was cut into small, $1 \mathrm{~mm}^{3}$ pieces and placed in a $6 \mathrm{~cm}^{2}$ culture dish. After adding $4 \mathrm{~mL}$ of DMEM medium and $500 \mu \mathrm{l}$ of collagenase type I (final concentration of $1 \mathrm{mg} / \mathrm{mL}$ ), the tissue was digested for $4 \mathrm{~h}$ in an incubator at $37^{\circ} \mathrm{C}$ and $5 \% \mathrm{CO}_{2}$. The tissue block was flocculent. The sample was filtered through a 100-mesh stainless steel filter and rinsed with PBS. The filtrate was centrifuged at $1500 \mathrm{rpm}$ for $5 \mathrm{~min}$, and the supernatant was discarded. The cells were resuspended in $15 \%$ FBS DMEM and placed in a $10-\mathrm{cm}^{2}$ culture dish, which was incubated at $37^{\circ} \mathrm{C}$ and $5 \% \mathrm{CO}_{2}$. After the confluence of the cells reached $>80 \%$, digestion and passage were performed. Thirdgeneration cells were digested with trypsin for the experiment.

\section{Effect of Drug-Carrying Serum on the Proliferation of FLSs In Vitro}

The third-generation osteoblasts were adjusted to a concentration of $2 \times 10^{3}$ cells $/ \mathrm{mL}$ and seeded in a 96-well culture plate at $100 \mu \mathrm{l} /$ well. After culture with medium containing $2 \%$ fetal bovine serum for 24 hours, the cells were divided into 4 groups:

a) The $20 \%$ blank serum group;

b) The $20 \%$ high-dose total flavonoids of Rhizoma Drynariae medicated serum group;

c) The $20 \%$ low-dose total flavonoids of Rhizoma Drynariae medicated serum group and

d) The $20 \%$ tripterygium glucosides medicated serum group, with 10 wells for each group.

After culture in an incubator at $37^{\circ} \mathrm{C}$ and $5 \% \mathrm{CO}_{2}$ for $24 \mathrm{~h}, 100 \mu \mathrm{l}$ of the culture solution was discarded and $20 \mu \mathrm{l}$ of thiazolyl tetrazolium (MTT) solution $(5 \mathrm{mg} / \mathrm{mL})$ was added to each well, followed by incubation at $37^{\circ} \mathrm{C}$ for $4 \mathrm{~h}$. The supernatant was discarded, and the crystals were dissolved in $150 \mu \mathrm{l}$ of dimethyl sulfoxide (DMSO) per well. The absorbance (wavelength at $490 \mathrm{~nm}$ ) was measured using an enzyme-linked immunosorbent assay (ELISA) analyzer. The calculation performed was as follows: cell viability $\%=(\mathrm{OD}$ of the tested well / OD of the control well) $\times 100 \%$.

Effect of Drug-Carrying Serum on the Expression of Proliferating Cell Nuclear Antigen (PCNA) mRNA and Bcl-2 mRNA in FLSs in vitro

a) The third-generation osteoblasts were digested with $0.25 \%$ trypsin to prepare a single cell suspension by repeated pipetting; the cell concentration was adjusted to $2 \times 104 / \mathrm{mL}$, and the cells were seeded onto a 12 -well cell culture plate in $1 \mathrm{~mL}$ per well. The cells were cultured in an incubator at $37^{\circ} \mathrm{C}$ and $5 \% \mathrm{CO}_{2}$ for $24 \mathrm{~h}$. The culture medium in the well was removed completely and replaced with culture medium containing $1 \%$ fetal bovine serum for $12 \mathrm{~h}$; then, the culture medium in the well plate was aspirated and replaced with $20 \%$ blank control serum, 20\% high-dose total flavonoids of Rhizoma Drynariae drug-carrying serum, 20\% lowdose total flavonoids of Rhizoma Drynariae drug-carrying serum or 
$20 \%$ of tripterygium glycosides drug-carrying serum, followed by culture for another $24 \mathrm{~h}$.

b) The total RNA of the FLSs was extracted following the instructions of the Trizol one-step reagent, and $1 \mu \mathrm{g}$ of total RNA was used as the template to synthesize cDNA by reverse transcription.

c) The primers were synthesized by Shanghai Shenggong Bioengineering Technology Service Co., Ltd.; the primer sequences are provided in Table 1.

d) Real-time quantitative PCR: the fluorescent dye SYBR Green and the primers for the target gene, Bcl-2, and the internal reference, actin, were mixed in $5 \mu$ l of SYBR Green $+0.15 \mu$ l of primers. After sealing, the reaction mixture was put into a real-time PCR analyzer (ABI 7900) for PCR to determine the CT values for PCNA and Bcl-2 mRNA expression. The expression level was calculated based on the $2^{-\Delta C \mathrm{~T}}$ equation (Table 1 ).

Statistical Analysis: Statistical analysis was performed on the experimental data using SPSS 18.0 software. The normally distributed data are presented as means \pm standard deviations. If the variance of each treatment group was homogeneous, the LSD-t test was used for the comparison between the two groups. When the mean values of the two groups of samples were compared, if the variance was homogeneous, the t-test was used; if the variance was heterogeneous, the $\mathrm{t}^{\prime}$ test was used. Differences with $\mathrm{P}<0.05$ were considered statistically significant.

Table 1: The genes detected by quantitative PCR and the corresponding primer sequences.

\begin{tabular}{|c|c|c|}
\hline Gene & Sequence from $\mathbf{5}^{\prime}$ to $\mathbf{3}^{\prime}$ & $\begin{array}{c}\text { Length of } \\
\text { Product (bp) }\end{array}$ \\
\hline Actin & $\begin{array}{c}\text { AGGCCAACCGTGAAAAGATG (forward) } \\
\text { ACCAGAGGCATACAGGGACAA (reverse) }\end{array}$ & 70 \\
\hline PCNA & $\begin{array}{c}\text { AAGCCGAAACCAGCTAGACTTTC (forward) } \\
\text { TGGCGGAGTGGCAACAA (reverse) }\end{array}$ & 71 \\
\hline Bcl-2 & $\begin{array}{c}\text { GGCATCTGCACACCTGGAT (forward) } \\
\text { AGACAGCCA GGAGAAATCAAACA (reverse) }\end{array}$ & 81 \\
\hline
\end{tabular}

\section{Results}

\section{Effects of the Serum Carrying Total Flavonoids of Rhizoma Drynariae on the in vitro Cultured FLSs}

Table 2: In vitro stimulation of RA-FLSs by treatment with $20 \%$ drug-carrying serum for $48 \mathrm{~h}$.

\begin{tabular}{|c|c|c|}
\hline Group & $\mathbf{n}$ & OD Value \\
\hline Blank control & 10 & $1.6387 \pm 0.0371$ \\
\hline $\begin{array}{c}\text { Total flavonoids of Rhizoma } \\
\text { Drynariae }\end{array}$ & & \\
\hline High dose & 10 & $0.9385 \pm 0.1517 \mathrm{a}^{* *}$ \\
\hline Low dose & 10 & $1.0815 \pm 0.3228 \mathrm{~b}^{* *}$ \\
\hline Tripterygium glucosides & 10 & $1.2873 \pm 0.0152 \mathrm{c}^{* *}$ \\
\hline
\end{tabular}

Note: Compared with the blank control group, $* * \mathrm{P}<0.0001$; ${ }^{a} t=11.325 ;{ }^{b}=6.125 ;{ }^{c}=6.335$.
As shown in Table 2, compared with the blank control group, treatment with sera containing 20\% high-dose and low-dose total flavonoids of Rhizoma Drynariae and tripterygium glycosides could significantly inhibit the proliferation of FLSs $(\mathrm{P}<0.0001)$, with no significant differences between the three groups (Table 2).

\section{Effect of Drug-Carrying Serum on the Expression of PCNA mRNA in the in vitro Cultured FLSs}

As shown in Table 3, compared with the blank control group, treatment with sera containing $20 \%$ high-dose and low-dose total flavonoids of Rhizoma Drynariae and tripterygium glycosides could significantly inhibit the expression of PCNA mRNA in FLS $(\mathrm{P}<0.0001)$, in which the inhibitory effect of the high-dose total flavonoids of Rhizoma Drynariae was more significant than that of the low-dose treatment $(\mathrm{P}=0.037)$.

Table 3: PCNA mRNA expression and BCL-2 mRNA expression.

\begin{tabular}{|c|c|c|c|}
\hline Group & $\mathbf{n}$ & $\begin{array}{c}\text { PCNA mRNA } \\
\text { Expression }\end{array}$ & $\begin{array}{c}\text { Bcl-2 mRNA } \\
\text { Expression }\end{array}$ \\
\hline Blank control & 10 & $19.6852 \pm 6.8975$ & $14.6961 \pm 7.5714$ \\
\hline $\begin{array}{c}\text { Total flavonoids of } \\
\text { Rhizoma Drynariae }\end{array}$ & & & $3.74051 \pm 2.2$ \\
\hline high dose & 10 & $412 \mathrm{a} 1$ & $3.7396 \pm 2.2303 \mathrm{a} 2$ \\
\hline low dose & 10 & $\begin{array}{c}6.5602 \pm 4.0215 \\
\mathrm{~b} 1\end{array}$ & $6.4510 \pm 4.0329 \mathrm{~b} 2$ \\
\hline Tripterygium glucosides & 10 & $\begin{array}{c}4.2176 \pm 1.8450 \\
\mathrm{c} 1\end{array}$ & $5.5108 \pm 4.3342 \mathrm{c} 2$ \\
\hline
\end{tabular}

Note: Compared with the blank control group, ${ }^{a 1} t=6.053$, $\mathrm{P}<0.0001 ; \quad{ }^{\mathrm{b}}{ }_{\mathrm{t}}=4.879, \quad \mathrm{P}=0.0004 ; \quad{ }^{\mathrm{c}} \mathrm{t}=4.745, \quad \mathrm{P}=0.0004 . \quad{ }^{\mathrm{a} 2} \mathrm{t}=5.785$, $\mathrm{P}<0.0001 ;{ }^{\mathrm{b} 2} \mathrm{t}=4.754, \mathrm{P}=0.0005 ;{ }^{\mathrm{c} 2} \mathrm{t}=4.753, \mathrm{P}=0.0005$.

\section{Effect of Drug-Carrying Serum on the Expression of Bcl-2 mRNA in the in vitro Cultured FLS}

As shown in Table 3, compared with the blank control group, treatment with sera containing $20 \%$ high-dose and low-dose total flavonoids of Rhizoma Drynariae and tripterygium glycosides could significantly inhibit the expression of Bcl-2 mRNA in FLS $(\mathrm{P}<0.0001)$, with no statistically significant differences between the three groups (Table 3).

\section{Conclusion}

Total flavonoids of Rhizoma Drynariae may inhibit the proliferation of FLSs by regulating the expression of the PCNA and Bcl-2 genes, thereby inhibiting inflammation and reducing bone loss.

\section{Discussion}

FLSs in patients with RA can play an important role in inflammation and joint destruction by secreting various proteases, arachidonic acid metabolites and their cytokines [4-6]. They are the ultimate target cells for the pathological changes of RA, and their excessive proliferation may result in synovial hyperplasia, which is one of the main pathological features of RA [7,8]. Currently, 
the hypothesis of insufficient apoptosis of FLS received the most attention. When the number of cells in inflammatory synovial tissue is multiplied, under normal conditions, the body can eliminate these excess cells through apoptosis to maintain a balance, but insufficient cell apoptosis may lead to hyperplasia of the synovial tissue and even a series of pathological changes in arthritis. Therefore, the excess hyperplasia and insufficient apoptosis of FLSs are the main mechanisms of RA and inhibiting the proliferation of FLSs and inducing their apoptosis are considered to be effective methods for treating RA [2,9]. Traditional plant extracts, such as celastrol [10] and curcumin [11], can inhibit the proliferation of FLSs by inducing DNA damage, cell cycle arrest and apoptosis; thus, they are considered to have potential in treating RA. However, their application is limited due to their high toxicity and lack of identification of the active ingredients.

The Chinese traditional drugs used in RA treatment have multiple targets and multiple pathways, with a wide range of pharmacological activities, showing the importance of their development and their research value. The continuous efficacy and safety of chemical synthetic drugs and kinase inhibitors in the treatment of RA were confirmed by a clinical study, but their longterm safety remains to be further investigated [12]. Proliferating cell nuclear antigen (PCNA) is an accessory protein of DNA polymerase 8 , also known as cyclin. As a peptide synthesized and expressed only in proliferating cells, PCNA plays an important role in the initiation of cell proliferation. The change in its content and expression level is consistent with the activity of DNA synthesis and replication, which can be used as an indicator to evaluate the state of cell proliferation. Our study showed that the high-dose and lowdose total flavonoids of Rhizoma Drynariae medicated sera could significantly inhibit the expression of PCNA mRNA in FLSs and that the effect of the high-dose treatment was more significant than that of the low-dose treatment. The Bcl-2 gene family, which is the apoptosis-regulating gene family that receives the most attention, and its related protein Bcl-2 are the earliest studied genes related to apoptosis.

$\mathrm{Bcl}-2$ is an important apoptosis-regulating gene that can prolong cell life by inhibiting various forms of cell death, leading to an increase in the number of cells and an increase in the mutation rate of genetic material. The high-dose and low-dose total flavonoids of Rhizoma Drynariae medicated sera could significantly inhibit the expression of Bcl-2 mRNA in FLSs. In this study, the serum pharmacological research method was used to observe the effect of serum carrying total flavonoids of Rhizoma Drynariae on the proliferation and apoptosis of FLSs in vitro. The results showed that $20 \%$ serum carrying total flavonoids of Rhizoma Drynariae could promote the proliferation of FLSs cultured in vitro and that the effect of the high-dose treatment was more significant than that of the low-dose treatment. It was further confirmed by the results of real-time quantitative PCR that the expression levels of PCNA and Bcl-2 were decreased significantly after treatment with the drug-carrying sera, indicating that the proliferation of FLSs was significantly inhibited, which is consistent with the results of the MTT assay. This may also be one of the mechanisms by which the serum carrying total flavonoids of Rhizoma Drynariae can downregulate the proliferation of FLSs. This study provides a powerful theoretical basis for the clinical treatment of RA using total flavonoids of Rhizoma Drynariae at the cellular and molecular levels.

\section{Declarations}

\section{Ethics Approval and Consent to Participate}

Ethical approval was given by the Ethics Committee of Guanghua Hospital Affiliated to Shanghai University of Traditional Chinese Medicine, Shanghai, China. All patients gave their written information consent.

\section{Consent for Publication}

Not applicable.

\section{Availability of Data and Material}

The datasets used and/or analysed during the current study are available from the corresponding author on reasonable request.

\section{Competing Interests}

All the authors declare that they have no conflict of interest.

\section{Funding}

This work was supported by the Project of Shanghai Science and Technology Commission, grant number 134119b1500 and the Natural Science Project of Shanghai University of Traditional Chinese Medicine, grant number 18LK077.

\section{Author's Contributions}

LS and XL contributed to the study design. All authors collected the data and performed the data analysis. All authors prepared the manuscript.

\section{Acknowledgements}

None.

\section{References}

1. Bartok B, Firestein GS (2010) Fibroblast-like synoviocytes: key effector cells in rheumatoid arthritis. Immunol Rev 233(1): 233-255.

2. Wang Fengjie, Fan Shasha, Chen Xianbing (2016) Effects of Lunasin on the proliferation and apoptosis of fibroblast-like synoviocytes in experimental rheumatoid arthritis rats. Chinese Journal of Immunology 32(2): 114-118.

3. Li Jinyu, Zhao Xueqian, Sun Qi (2018) Basic and clinical research of total flavonoids from drynaria fortunei [J]. Chinese Journal of Osteoporosis 24(10): 1357-1363.

4. Zhou Zhenhua, Xu Weidong, Wu Yuesong (2008) Progress in the correlation study of fibroblast-like synoviocytes and rheumatoid arthritis. Chinese Journal of Joint Surgery 2(6): 52-55.

5. Yamanishi Y, Firestein GS (2001) Pathogenesis of rheumatoid arthritis: the role of synoviocytes. Rheum Dis Clin North Am 27(2): 355-371. 
6. Fox DA, Gizinski A, Morgan R, Lundy SK (2010) Cell-cell interactions in rheumatoid arthritis synovium. Rheum Dis Clin North Am 36(2): 311323.

7. Huang Xueying, Chen Feihu, Zhang Xiaoming (2009) Effects of recombinant human endostatin on the cell cycle and PCNA expression in fibroblast-like synoviocytes of rats with adjuvant arthritis. Chinese Pharmacological Bulletin 25(5): 649-653.

8. Nishioka K, Hasunuma T, Kato T, Sumida T, Kobata T (1998) Apoptosis in rheumatoid arthritis: a novel pathway in the regulation of synovial tissue. Arthritis Rheum 41(1): 1-9

9. Pope RM (2002) Apoptosis as a therapeutic tool in rheumatoid arthritis Nat Rev Immunol 2(7): 527-535.

\section{ISSN: 2574-1241}

DOI: 10.26717/BJSTR.2019.21.003595

Shaohua Li, Lianbo Xiao. Biomed J Sci \& Tech Res

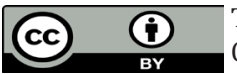

This work is licensed under Creative Commons Attribution 4.0 License

Submission Link: https://biomedres.us/submit-manuscript.php
10. Xu Z, Wu G, Wei X, Chen X, Wang Y, et al. (2013) Celastrol induced DNA damage, cell cycle arrest, and apoptosis in human rheumatoid fibroblastlike synovial cells. Am J Chin Med 41(3): 615-628.

11. Qiu Yan, Shang Wei, Zhao Zhiming (2014) Effect of curcumin on the expression of synovial osteoprotegerin and receptor activator of nuclear factor-кB ligand protein in adjuvant arthritis rats. Chinese Journal of Immunology 30(11): 1490-1494.

12. Wang Yongzhong, Deng Longfei, Han Yanquan (2016) Primary culture of fibroblast-like synoviocytes and its application in the research of therapeutic mechanisms of rheumatoid arthritis. China Pharmacy 27(7): 966-968.

BIOMEDICAL
RESEARCHES $\quad$\begin{tabular}{l} 
Assets of Publishing with us \\
\hline
\end{tabular}

